\title{
Design of Frequency Invariant Beamformer for Broadband Arrays
}

\author{
Wei Liu \\ Communications Research Group \\ Dept. of Electronic \& Electrical Engineering \\ University of Sheffield, U.K.
}

\author{
Stephan Weiss \\ Institute of Comms \& Signal Processing \\ School of Electronic \& Electrical Engineering \\ University of Strathclyde, U.K.
}

\begin{abstract}
A simple method for the design of a class of arrays with frequency invariant beam patterns is proposed. Starting from the desired frequency invariant beam pattern of an $n$-D array, the proposed method uses a series of substitutions and an $n$-D inverse Fourier transform to obtain the desired frequency responses of the filters following each sensor. Given their desired frequency responses, these filters can be realized by either an analogue filter or a digital filter. Hence the proposed method can cover the design of broadband arrays with either analogue signals or discrete signals. Two design examples are provided, with one for a linear array and one for a planar array.
\end{abstract}

\section{INTRODUCTION}

In the past, broadband beamformers have been studied extensively due to their applications to sonar, radar, communications [1], [2]. Amongst them is a class of arrays with frequency invariant responses [3], [4], [5], [6], [7], which can achieve a beam pattern independent of frequency, therefore with a constant beamwidth.

The design of a frequency invariant beamformer can be achieved by direct optimization for all of the coefficients with respect to the desired response [8], but for large linear/planar arrays, the number of coefficients to be optimized will be extremely large. Therefore a low-complexity systematic method is needed for the design of frequency invariant beamformers. One of such methods was proposed in [6], where each element of the array is followed by its own primary filter and the outputs of these primary filters share a common secondary filter to form the final output. However, although the design for a 1-D array is relatively simple because of the dilation property of the primary filters, for higher-dimensional arrays this property is not guaranteed, which makes the general design case too complicated and no design examples for a 2-D array were provided there. Recently, another systematic method was proposed in [9]. By exploiting the Fourier transform relationship between the array's spatial and temporal parameters and its beam pattern, two parallel theories/design methods for frequency invariant beamforming were proposed for continuous sensor array with analogue signals and discrete sensor array with discrete signals, respectively. With the proposed methods, the design of higher-dimensional arrays can be implemented by simple Fourier transforms.

However, there is a gap between the two parallel theories/design methods, i.e. for the case with discrete sensors and analogue signals. In this paper, we aim to fill this gap by dealing with the design of discrete sensors with analogue signals and the proposed method for discrete sensors with discrete signals in [9] can be considered as a special case. Starting from the desired frequency invariant beam pattern of an $n$-D array, the proposed method uses a series of substitutions and an $n$-D inverse Fourier transform to achieve a frequency invariant beamformer design. The proposed method is general as it covers the case of discrete sensors with either analogue signals or discrete signals.

The paper is organised as follows. Section II addresses the design problem with continuous sensors first, then the case with discrete sensor arrays is discussed as an approximation to the continuous case. Thereafter a systematic design procedure is derived. Two design examples - one for a linear array and one for a planar array — are given to highlight its effectiveness in Section III and conclusions are drawn in Section IV.

\section{FREQUENCY INVARIANT BEAMFORMING}

\section{A. One-dimensional Array}

Fig. 1 (a) shows a 1-D continuous sensor array aligned with the $x$ axis. The beam response of this linear array is given by

$$
P(\omega, \theta)=\int_{-\infty}^{\infty} e^{-j \frac{\omega \sin \theta}{c} x} D(x, \omega) d x,
$$

where $c$ and $\theta$ are the propagation speed and angle of the impinging signal, respectively, and $D(x, \omega)$ is the frequency response of the sensor at location $x$ with respect to the angular frequency $\omega$. With the substitution $\omega_{1}=\frac{\omega \sin \theta}{c}$, we have

$$
P\left(\omega_{1}, \omega\right)=\int_{-\infty}^{\infty} e^{-j \omega_{1} x} D(x, \omega) d x .
$$

Clearly $P(\omega, \theta)$ can be obtained by first applying a 1-D Fourier transform to $D(x, \omega)$ and then re-substituting $\omega_{1}=\frac{\omega \sin \theta}{c}$.

For frequency invariant beam response, $P\left(\omega_{1}, \omega\right)$ must be a function of only $\theta$, or more precisely $\sin \theta$. Let $F(\sin \theta)$ be such a frequency invariant response. In order to match this desired beam pattern, $P\left(\omega_{1}, \omega\right)$ must, after re-substituting $\omega_{1}=\frac{\omega \sin \theta}{c}$, be identical to $F(\sin \theta)$. To achieve this, $\omega_{1}$ and $\omega$ must obey a specific dependency in the expression of $P\left(\omega_{1}, \omega\right)$ for $\omega$ to disappear. Note that if $P\left(\omega_{1}, \omega\right)$ is a function of $c \frac{\omega_{1}}{\omega}$, since $c \frac{\omega_{1}}{\omega}=c \frac{\omega \sin \theta}{c \omega}=\sin \theta$, it will eliminate any dependency on 


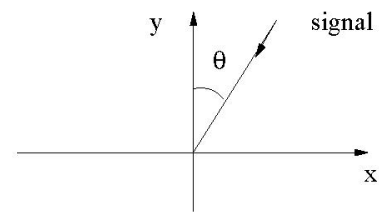

(a)

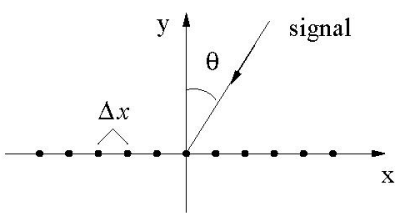

(b)

Fig. 1. (a). A continuous sensor array aligned with the $x$ axis; (b). An equally spaced linear array with a sensor spacing of $\Delta x$.

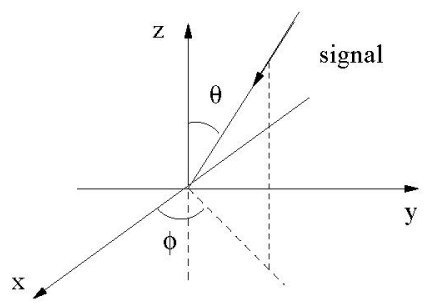

(a)

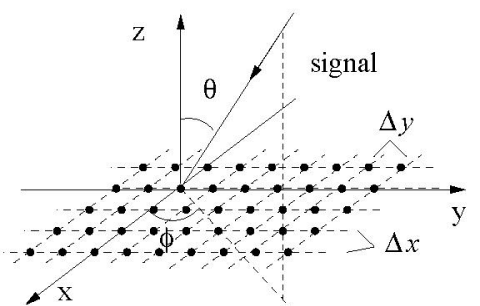

(b)

Fig. 2. (a). A continuous 2-D sensor array; (b). An equally spaced planar array with sensor spacings of $\Delta x$ and $\Delta y$ respectively.

$\omega$. Therefore, we can set $P\left(\omega_{1}, \omega\right)=F\left(\frac{\omega_{1} c}{\omega}\right)$ for the desired response $F(\sin \theta)$. Applying an inverse Fourier transform to $P\left(\omega_{1}, \omega\right)$ with respect to the variable $\omega_{1}$, we obtain the desired frequency response $D(x, \omega)$ for any position $x . D(x, \omega)$ can then be realized by either an analogue filter or a digital filter, with an appropriate filter design method.

However, in practice, normally we only have sensors at specific positions. Suppose the sensor positions are given by $x=\ldots,-\Delta x, 0, \Delta x, \ldots$, as shown in Fig. 1 (b). Then the integration in (1) changes to summation:

$$
P(\omega, \theta)=\sum_{m=-\infty}^{\infty} D(m \Delta x, \omega) e^{-j \frac{\omega \sin \theta m \Delta x}{c}} .
$$

With $\omega_{1}=\frac{\omega \sin \theta \Delta x}{c}$, we have

$$
P\left(\omega_{1}, \omega\right)=\sum_{m=-\infty}^{\infty} D(m \Delta x, \omega) e^{-j m \omega_{1}}
$$

In order to avoid aliasing, $\Delta x<\lambda_{\min } / 2$, where $\lambda_{\min }$ is the wavelength of the maximum frequency of interest $\omega_{\max }$. Based on the discussion in the continuous sensor case, the design of this discrete sensor case can be described as follows.

Step 1. Given a desired frequency invariant beam pattern $F(\sin \theta)$, and the substitution $\sin \theta=\frac{\omega_{1} c}{\omega \Delta x}$,

$$
P\left(\omega_{1}, \omega\right)=F\left(\frac{\omega_{1} c}{\omega \Delta x}\right)
$$

is obtained for $\omega_{1} \in[-\pi ; \pi)$. As the Fourier transform of $D(m \Delta x, \omega), P\left(\omega_{1}, \omega\right)$ should be a periodic function with a period of $2 \pi$. For $\omega_{1} \in[-\pi ; \pi)$, as there is no signal existing for both $\left|\omega_{1}\right|>\left|\frac{\omega \Delta x}{c}\right|$ and $\omega=0$, the value of $P\left(\omega_{1}, \omega\right)$ in this area can be modified arbitrarily.

Step 2. Applying the 1-D inverse Fourier transform (IFT) to $P\left(\omega_{1}, \omega\right)$ with respect to $\omega_{1}$, we can get the desired frequency responses $D(m \Delta x, \omega)$ for the sensor at a position of $m \Delta x, m=\ldots,-1,0,1, \ldots$ Suppose there are $M$ sensors in total. $D(m \Delta x, \omega)$ can be obtained by the inverse discrete Fourier transform (IDFT) as an approximation, by sampling $P\left(\omega_{1}, \omega\right)$ on the $\tilde{M}>M$ points of $\omega_{1}=-\pi+\frac{2 \tilde{m} \pi}{\tilde{M}}, \tilde{m}=0,1, \ldots, \tilde{M}-1$. Then $D(\tilde{m} \Delta x, \omega)$ can be obtained easily by the $\tilde{M}$-point IDFT of $P\left(-\pi+\frac{2 \tilde{m} \pi}{\tilde{M}}, \omega\right)$. To fit the real dimension $M$ of the array, we need to truncate the resultant $D(m \Delta x, \omega)$ to the size of $M$, with an appropriate window function, such as a rectangular window or a hamming window.

The approach outlined above can be applied to any desired frequency invariant beam pattern $F(\sin \theta)$.

\section{B. Two-dimensional and Three-dimensional Arrays}

Fig. 2 (a) shows a 2-D continuous sensor array in the $(x, y)$ plane. The beam response of this continuous array is given by

$$
P(\omega, \theta, \phi)=\iint_{\infty}^{\infty} e^{-j \frac{\omega}{c}(x \sin \theta \cos \phi+y \sin \theta \sin \phi)} D(x, y, \omega) d x d y
$$

where $D(x, y, \omega)$ is the frequency response of the sensor at the point $(x, y)$. The substitutions $\omega_{1}=\frac{\omega \sin \theta \cos \phi}{c}$ and $\omega_{2}=$ $\frac{\omega \sin \theta \sin \phi}{c}$ into (6) results in

$$
P\left(\omega_{1}, \omega_{2}, \omega\right)=\iint_{\infty}^{\infty} D(x, y, \omega) e^{-j \omega_{1} x} e^{-j \omega_{2} y} d x d y
$$


i.e. $P\left(\omega_{1}, \omega_{2}, \omega\right)$ is the 2-D Fourier transform of $D(x, y, \omega)$. If this Fourier transfrom can be expressed as $P\left(\omega_{1}, \omega_{2}, \omega\right)=$ $F\left(\frac{\omega_{1} c}{\omega}, \frac{\omega_{2} c}{\omega}\right)$, where $F\left(\hat{\omega}_{1}, \hat{\omega}_{2}\right)$ is the frequency response of a 2-D filter with $\hat{\omega}_{1}$ and $\hat{\omega}_{2}$ representing the two corresponding frequency variables, then the resulting function $F(\sin \theta \cos \phi, \sin \theta \sin \phi)$ will represent the frequency invariant beam pattern.

Alternatively, suppose $F(\sin \theta \cos \phi, \sin \theta \sin \phi)$ is the desired beam pattern. We set $P\left(\omega_{1}, \omega_{2}, \omega\right)=F\left(\frac{\omega_{1} c}{\omega}, \frac{\omega_{2} c}{\omega}\right)$. Applying a 2-D inverse Fourier transform to $P\left(\omega_{1}, \omega_{2}, \omega\right)$ with respect to the variables $\omega_{1}$ and $\omega_{2}$, we then obtain the desired frequency response $D(x, y, \omega)$ for any position $(x, y)$.

Now consider the uniformly spaced discrete sensor case. Suppose the sensors are positioned at the locations of $(l \Delta x, m \Delta y)$, $l, m=\ldots,-1,0,1, \ldots$, as shown in Fig. 2 (b). Then (6) changes to

$$
\begin{gathered}
P(\omega, \theta, \phi)=\sum_{l, m=-\infty}^{\infty} D(l \Delta x, m \Delta y, \omega) e^{-j \frac{\omega l(l \Delta x \sin \theta \cos \phi+m \Delta y \sin \theta \sin \phi)}{c}} \text { and } \\
P\left(\omega_{1}, \omega_{2}, \omega\right)=\sum_{l, m=-\infty}^{\infty} D(l \Delta x, m \Delta y, \omega) e^{-j l \omega_{1}} e^{-j m \omega_{2}}
\end{gathered}
$$

with $\omega_{1}=\frac{\omega \sin \theta \cos \phi \Delta x}{c}$ and $\omega_{2}=\frac{\omega \sin \theta \sin \phi \Delta y}{c}$. To avoid aliasing, $\Delta x, \Delta y<\lambda_{\min } / 2$. Analogous to the 1-D design in Sec. II-A, the 2-D frequency invariant beamformer can be designed as follows.

Step 1. With the substitutions $\sin \theta \cos \phi=\frac{c \omega_{1}}{\omega \Delta x}$ and $\sin \theta \sin \phi=\frac{c \omega_{2}}{\omega \Delta y}$, we obtain $P\left(\omega_{1}, \omega_{2}, \omega\right)$ defined over one period $\omega_{1}, \omega_{2} \in[-\pi ; \pi)$ as

$$
P\left(\omega_{1}, \omega_{2}, \omega\right)=\left\{\begin{array}{ll}
F\left(\frac{c \omega_{1}}{\omega \Delta x}, \frac{c \omega_{2}}{\omega \Delta y}\right) & \text { for } 0<\left|\omega_{1}\right| \leq\left|\frac{\omega \Delta x}{c}\right| \wedge 0<\left|\omega_{2}\right| \leq\left|\frac{\omega \Delta y}{c}\right| \\
A\left(\omega_{1}, \omega_{2}\right) & \text { for otherwise }
\end{array},\right.
$$

where $A\left(\omega_{1}, \omega_{2}\right)$ is an arbitrary function with finite values, which will not affect the beam pattern as there is no signal in the area defined for it.

Step 2. Applying the 2-D IFT to $P\left(\omega_{1}, \omega_{2}, \omega\right)$ with respect to $\omega_{1}$ and $\omega_{2}$ returns the desired frequency response $D(l \Delta x, m \Delta y, \omega)$ for the corresponding sensors. Again the result can be obtained by the 2-D IDFT as an approximation, as described in the 1-D array case.

For 3-D arrays, as in [9], its design is analogous to those in Secs. II-A and II-B, and therefore omitted here.

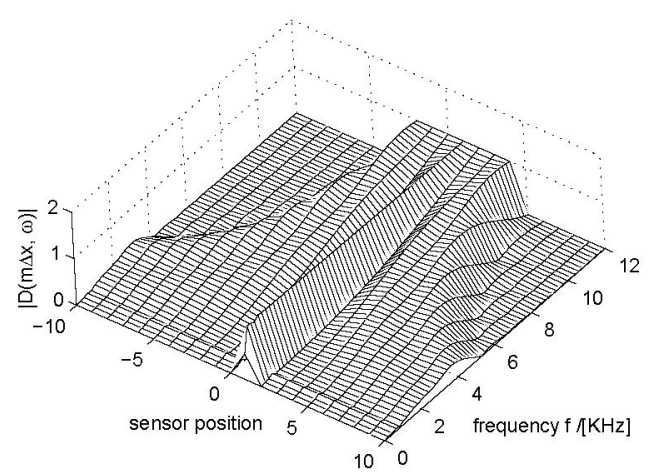

Fig. 3: The desired magnitude responses $|D(m \Delta x, \omega)|$ of the 21 filters for the linear array design.

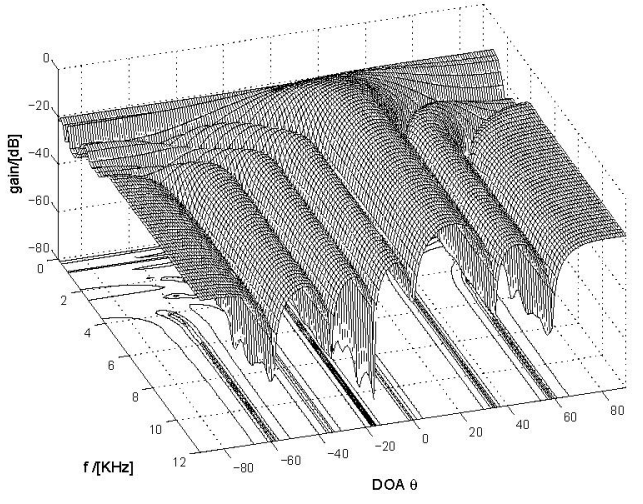

Fig. 4: The resultant frequency invariant beam pattern for the linear array design.

\section{DESIGN EXAMPLES}

We provide two examples for the design of a frequency invariant beamformer with potential applications to microphone arrays. The maximum frequency of interest is set to be $12 \mathrm{KHz}$ and the propagation speed is $340 \mathrm{~m} / \mathrm{s}$. The first example is for a linear array with 21 sensors and the second one for a planar array with $21 \times 21$ sensors, both with an adjacent sensor spacing of $34000 /(2 \times 12000)=1.42 \mathrm{~cm}$. The desired beam pattern for the linear array is given by

$$
F_{1 D}(\sin \theta)=\frac{1}{5} \sum_{m=-2}^{2} e^{-j m \pi(\sin \theta-0.2857)}
$$

which forms a main beam with a zero response to the broadside.

We employed a 64-point 1-D IDFT on the resultant function $P\left(\omega_{1}, \omega\right)$ and set $P\left(\omega_{1}, \omega\right)=0$ for the area where there is no signal existing, as indicated in Step 1 of the 1-D array design. The resultant 64 desired frequency responses $D(m \Delta x, \omega)$, with each corresponding to an analogue or digital filter following each of the 21 sensors, were then truncated to the size of 21 directly, with a rectangular window. Given the 21 desired frequency responses $D(m \Delta x, \omega), m=-10, \ldots, 1,0,1, \ldots, 10$, 
we can employ an appropriate filter design method to design 21 analogue or digital filters to realize them. The magnitude responses $|D(m \Delta x, \omega)|$ are shown in Fig. 3 to give a rough idea about the design results. As the filter design problem is not the focus of this paper, we assumed that the 21 filters had been obtained with the desired frequency responses $D(m \Delta x, \omega)$ (the same assumption for the planar array design) and then used $D(m \Delta x, \omega)$ to calculate the resultant beam pattern, which is shown in Fig. 4 with a clear frequency invariant property except for very low frequencies.

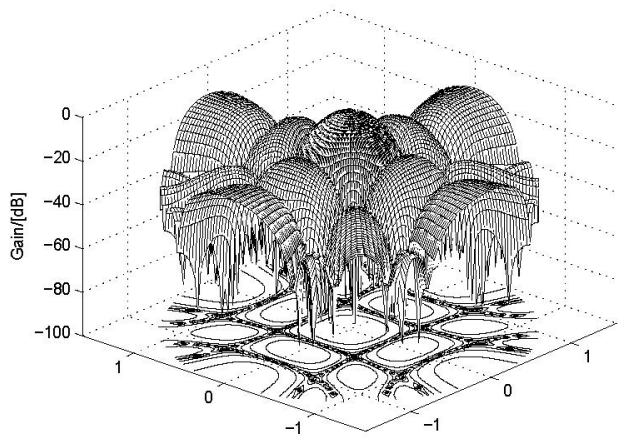

Fig. 5: The resultant beam pattern at $f=7.2 \mathrm{KHz}$

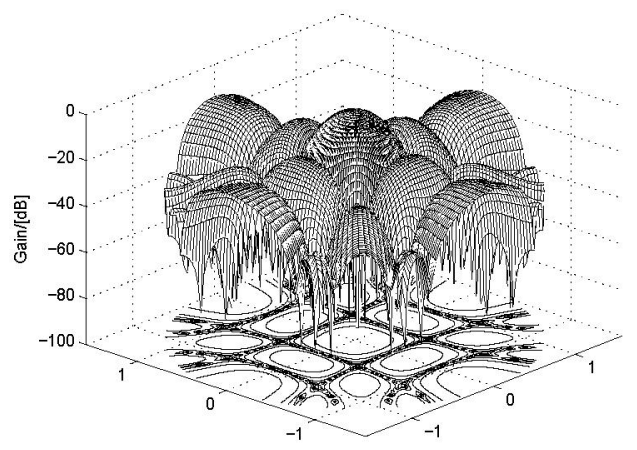

Fig. 6: The resultant beam pattern at $f=9.6 \mathrm{KHz}$.

For the second design example, the desired beam pattern is given by

$$
F_{2 D}(\sin \theta \cos \phi, \sin \theta \sin \phi)=\frac{1}{25} \sum_{l=-2}^{2} \sum_{m=-2}^{2} e^{-j l \pi \sin \theta \cos \phi} e^{-j m \pi \sin \theta \sin \phi} .
$$

According to (10), we first obtained the response $P\left(\omega_{1}, \omega_{2}, \omega\right)$ with $A\left(\omega_{1}, \omega_{2}\right)=0$. A $64 \times 642$-D IDFT was employed and the $64 \times 64$ desired frequency responses $D(l \Delta x, m \Delta y, \omega)$ were then subsequently truncated to the intended array dimension of $21 \times 21$. The resultant beam pattern is four-dimensional and here we can only provide some exemplary snapshots. Figs. 5 and 6 are the array's response in a cylindrical coordinates to the frequencies $f=7.2 \mathrm{KHz}$ and $f=9.6 \mathrm{KHz}$, respectively. The height axis is the magnitude response of the beam, the radial coordinate is for the elevation angle $\theta$ and the angle coordinate is for the azimuth angle $\phi$. The frequency invariant property can be verified by the similarity of these two figures, and further shown by a slice of its beam pattern at $\phi=60^{\circ}$, as given in Fig. 7 .

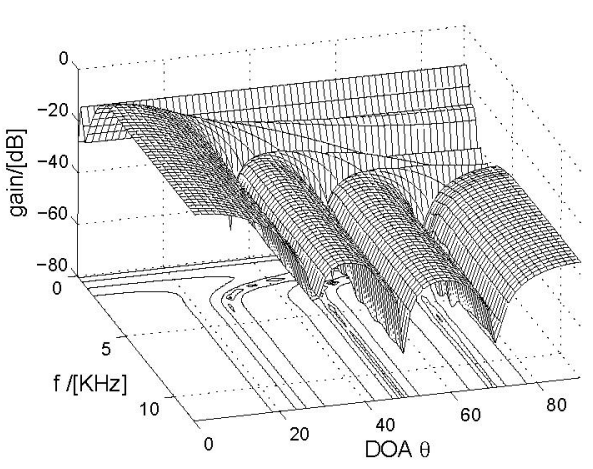

Fig. 7: A slice of the beam pattern at $\phi=60^{\circ}$.

\section{CONCLUSIONS}

We have developed a simple method for the design of a class of broadband arrays with frequency invariant beam patterns. By this method we obtain the desired frequency response of the filter following each sensor. With the desired frequency responses, these filters following array sensors can then be realized by either an analogue filter or a digital filter. Therefore the proposed method can cover the design of broadband arrays with either analogue signals or discrete signals. Two design examples have been provided, with one for a linear array and one for a planar array, yielding satisfactory frequency invariant characteristics over a large range of frequencies.

\section{REFERENCES}

[1] H. L. Van Trees, Optimum Array Processing, Part IV of Detection, Estimation, and Modulation Theory, John Wiley \& Sons, Inc., New York, U.S.A., 2002.

[2] M. Ghavami, L. B. Michael, and R. Kohno, Ultra Wideband Signals and Systems in Communication Engineering, John Wiley \& Sons, Ltd, Chichester, England, 2004.

[3] E. L. Hixson and K. T. Au, "Widebandwidth constant beamwidth acoustic array," Journal of the Aconstic Society of America, vol. 48, no. 1, pp. 117, July 1970.

[4] M. M. Goodwin and G. W. Elko, "Constant beamwidth beamforming," in Proc. IEEE International Conference on Acoustics, Speech, and Signal Processing, Minneapolis, USA, April 1993, vol. 1, pp. 169-172.

[5] T. Chou, "Frequency-independent beamformer with low response error," in Proc. IEEE International Conference on Acoustics, Speech, and Signal Processing, Detroit, USA, May 1995, vol. 5, pp. 2995-2998.

[6] D. B. Ward, R. A. Kennedy, and R. C. Williamson, "Theory and design of broadband sensor arrays with frequency invariant far-field beam patterns," Journal of the Acoustic Society of America, vol. 97, no. 2, pp. 1023-1034, February 1995.

[7] S. C. Chan and K. S. Pun, "On the design of digital broadband beamformer for uniform circular array with frequency invariant characteristics," in Proc. IEEE International Symposium on Circuits and Systems, Phoenix, USA, May 2002, vol. 1, pp. 693-696.

[8] S.F. Yang and Y. L. Ma, "Design of fir beamformer with frequency invariant patterns via jointly optimizing spatial and frequency responses," in Proc IEEE International Conference on Acoustics, Speech, and Signal Processing, Philadelphia, USA, March 2005, pp. 789-792.

[9] W. Liu and S. Weiss, "A new class of broadband arrays with frequency invariant beam patterns," in Proc. IEEE International Conference on Acoustics, Speech, and Signal Processing, Montreal, Canada, May 2004, vol. 2, pp. 185-188. 